\title{
Implementation of an A-stable block hybrid method for the solution of Bazykin ecological model and Trambouze reactor model
}

\author{
O Adedire ${ }^{1}, U . W$ Sirisena $^{2}$ and J. N Ndam ${ }^{3}$ \\ 1,2,3 Department of Mathematics, University of Jos, Nigeria \\ ${ }^{1}$ Federal College of Forestry, Jos, Plateau State, Nigeria
}

Received: 24 September 2019, Accepted: 29 April 2020

Published online: 22 June 2020.

\begin{abstract}
This research work inspects the appropriateness of a new block hybrid method with step number $\mathrm{k}=2$ incorporating an off-step collocation point for isothermal Trambouze reactor model and Bazykin ecological model with appropriate initial conditions. The newly derived A-stable block method proved effective for the solution of non stiff and stiff initial value problems of ordinary differential equations. Solutions using this block method indicate that the newly derived method competes satisfactorily with the inbuilt Matlab ODE solvers, ODE 15s and ODE 23s which have been acknowledged to solve similar problems effectively.
\end{abstract}

Keywords: A-stable, block hybrid method, multistep collocation, continuous formulation, absolute stability, isothermal Trambouze reaction, Bazykin, prey, predator, model.

\section{Introduction}

The design of chemical processes is targeted at the maximum yield operation. The research in [12] proposed an approach based on kinetic perception that optimizes the product distribution obtained from competing chemical reactions taking place in parallel. One of such reactions is the isothermal Trambouze reaction scheme which can be found in [17]. Trambouze reaction scheme consists of three parallel reactions and four chemical species described by

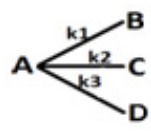

The reactor is loaded initially with pure A. For this reaction scheme, the desired product is C while B and D are by-products. The main objective was to maximize the selectivity related to species $\mathrm{C}$ and to minimize the total volume requirements. The selectivity is given by

$$
\frac{C_{c}}{1-C_{A}}
$$

\footnotetext{
*Corresponding author e-mail: dharenss@gmail.com
} 
where $C_{C}$ and $C_{A}$ are mole fractions of components $\mathrm{C}$ and $\mathrm{A}$ respectively. The reaction kinetics led to the following system of differential equations:

$$
\left.\begin{array}{l}
\frac{d\left(C_{A}\right)}{d t}=-k_{1}-k_{2} C_{A}-k_{3} C_{A}^{2} \\
\frac{d\left(C_{B}\right)}{d t}=k_{1} \\
\frac{d\left(C_{c}\right)}{d t}=k_{2} C_{A} \\
\frac{d\left(C_{D}\right)}{d t}=k_{3} C_{A}^{2}
\end{array}\right\}
$$

where $C_{j}(\mathrm{j}=\mathrm{A}, \mathrm{B}, \mathrm{C}, \mathrm{D})$ are molar concentration of components A,B,C and D respectively while $k_{j}(\mathrm{j}=1,2,3)$ are reaction rate constants.

Isothermal Trambouze reaction scheme is noted for having multiple solutions and it was stated in [12] that the solutions are infinitely many. The model (2) is a nonlinear complex chemical process which has been examined by some researchers. The research of [19] examined the system (2) only for pure A and product $\mathrm{C}$ as

$$
\left.\begin{array}{l}
\frac{d\left(C_{A}\right)}{d t}=-k_{1}-k_{2} C_{A}-k_{3} C_{A}^{2} \\
\frac{d\left(C_{c}\right)}{d t}=k_{2} C_{A}
\end{array}\right\}
$$

The system of equations presented in [4] which was further extensively studied in [6] is the system below:

$$
\left.\begin{array}{l}
\frac{d u}{d t}=a u-\frac{b u v}{1+\alpha u}-\in u^{2} \\
\frac{d v}{d t}=-c v+\frac{d u v}{1+\alpha u}-k v^{2}
\end{array}\right\}
$$

where $\mathrm{u}$ and $\mathrm{v}$ represent prey density and predator density respectively, the constant parameters $a, \mathrm{~b}, \alpha, \in, \mathrm{c}, \mathrm{d}, \mathrm{k}$ describe the behaviour of isolated populations and their interactions such that $a u$ describe the exponential growth of the prey population in the absence of predators.

The terms $-\epsilon u^{2}$ and $-k u^{2}$ are the intraspecific competition among prey and predator respectively while the term $-c v$ is the exponential reduction in the predator population in the absence of prey with $-\frac{b u v}{1+\alpha u}$ and $\frac{d u v}{1+\alpha u}$ describing the interaction between the two populations [18].

It was stated in [10] that two forms of predator-prey models exist with one as prey dependent and the other as ratio dependent systems where the ratio-dependent was studied by [10] for the cases where predators are involved in serious hunting. The emphasis here is on prey-dependent model as discussed in [6]. Detailed biological importance of classical prey-dependent and ratio-dependent models is available in [5]. Some researchers have solved similar models using ODE solvers such as MATLAB ODE 45, ODE 15 s and so on.

In this paper, we discuss a new A-stable block hybrid method with $x=x_{n+\frac{9}{5}}$ as an off-step collocation point for the numerical solution of the system (2) and system (4). Similar approach used in [1] produced an A- $(\alpha)$ stable method which does not have the benefit of A-stability obtained in this method. The choice of the $x=x_{n+\frac{9}{5}}$ as off-step point is such that it is close to the end point of the interval. We then compare the accuracy of our new method with MATLAB ODE $15 \mathrm{~s}$ and ODE $23 \mathrm{~s}$ which have been tested on numerous similar problems.

The subsequent part of this paper is organized as follows: section 2 deals with the derivation of the new block hybrid method incorporating $x=x_{n+\frac{9}{5}}$ as off-step collocation point. Section 3 deals with analysis of the new method. While in sections 4 and 5, the simulation and discussion of the models (2) and (4) will be done using the new method in 
comparison with the ODE 15 s and ODE 23s. Conclusion comes up in section 6.

\section{Derivation}

Using the notion of continuous finite difference approximation method of [13], and the multistep collocation approach put forward by [14] and [15], we define the continuous formulation of the k-step hybrid method as

$$
y(x)-\sum_{j=0}^{t-1} \alpha_{j}(x) y_{n+j}=h \beta_{v}(x) f_{n+v}+h \beta_{k}(x) f_{n+k}, \quad x_{n} \leq x \leq x_{n+k}
$$

where $\alpha_{j}(x), \beta_{v}(x)$ and $\beta_{k}(x)$ are continuous coefficients of the method, $\mathrm{t}$ is the number of distinct interpolation points $x_{n+j}, j=0, \ldots, t-1, x_{n+v}$ is an off-step collocation point, $v \notin\{0,1, \ldots, \mathrm{k}\}, \mathrm{f}_{n+v}=f\left(x_{n+v}, y_{n+v}\right)$ is an off-step function evaluation and $\mathrm{h}$ is the step size.

For parameters $\mathrm{k}=2, \mathrm{t}=2, \mathrm{~m}=2$, and $\mathrm{v}=\frac{9}{5}$, where $\mathrm{m}$ is the number of collocation points, (5) becomes

$$
y(x)=\alpha_{o}(x) y_{n}+\alpha_{1}(x) y_{n+1}+h \beta_{\frac{9}{5}}(x) f_{n+\frac{9}{5}}+h \beta_{2}(x) f_{n+2}
$$

Based on the multistep collocation derived and expounded in [16] and [17], we obtain matrix D as

$$
D=\left[\begin{array}{cccc}
1 & x_{n} & x_{n}^{2} & x_{n}^{3} \\
1 & x_{n+1} & x_{n+1}^{2} & x_{n+1}^{3} \\
0 & 1 & 2 x_{n+\frac{9}{5}} & 3 x_{n+\frac{9}{5}}^{2} \\
0 & 1 & 2 x_{n+2} & 3 x_{n+2}^{2}
\end{array}\right]
$$

and using matrix inversion technique as derived in the work done by [15], the continuous coefficients $\alpha_{o}(x), \alpha_{1}(x), \beta_{\frac{9}{5}}(x)$ and $\beta_{2}(x)$ of the continuous scheme (6) are obtained with the assistance of Maple software, which when substituted into (6) gives

$$
\begin{aligned}
& y(x)=\left\{\frac{1}{h^{3}}\left(h^{2}-\frac{47 h}{61}\left(x-x_{n}\right)+\frac{10}{61}\left(x-x_{n}\right)^{2}\right)\left(h-\left(x-x_{n}\right)\right)\right\} y_{n}+\left\{\frac { 1 0 8 } { 6 1 h ^ { 3 } } \left(h^{2}-\frac{19 h}{36}\left(x-x_{n}\right)\right.\right. \\
& \left.+\frac{5}{54}\left(x-x_{n}\right)^{2}\left(x-x_{n}\right)\right\} y_{n+1}+\left\{-\frac{25}{61 h^{2}}\left(\left(h-\left(x-x_{n}\right)\right)\left(8 h-3\left(x-x_{n}\right)\left(x-x_{n}\right)\right)\right)\right\} f_{n+\frac{9}{5}} \\
& \left.+\frac{1}{61 h^{2}}\left(\left(h-\left(x-x_{n}\right)\right)\left(153 h-65\left(x-x_{n}\right)\left(x-x_{n}\right)\right)\right)\right\} f_{n+2}
\end{aligned}
$$

Evaluating (8) at $x=x_{n+1}, x_{n+\frac{9}{5}}$ and $x_{n+2}$, we obtain the three discrete schemes below which shall be used as block integrator:

$$
\left.\begin{array}{l}
y_{n+1}=y_{n}+\frac{61}{24} h f_{n+1}-\frac{125}{24} h f_{n+\frac{9}{5}}+\frac{11}{3} h f_{n+2} \\
y_{n+\frac{9}{5}}=-\frac{176}{1525} y_{n}+\frac{1701}{1525} y_{n+1}+\frac{468}{305} h f_{n+\frac{9}{5}}-\frac{1296}{1525} h f_{n+2} \\
y_{n+2}=-\frac{7}{61} y_{n}+\frac{68}{61} y_{n+1}+\frac{100}{61} h f_{n+\frac{9}{5}}-\frac{46}{61} h f_{n+2}
\end{array}\right\}
$$




\section{Analysis of the new block method}

We take into account the convergence and plot of region of absolute stability of the newly assembled block hybrid method in this section.

\subsection{Zero Stability Analysis}

Using the approach found in[7], the discrete schemes (9) can be in block form as

$$
M^{(1)} Y_{n+i}=M^{(0)} Y_{n-i}+h B^{(1)} f_{n+i}, \quad i \in \mathbb{R}
$$

where

$$
M^{(1)}=\left(\begin{array}{ccc}
1 & 0 & 0 \\
-\frac{1701}{1525} & 1 & 0 \\
-\frac{68}{61} & 0 & 1
\end{array}\right), M^{(0)}=\left(\begin{array}{ccc}
0 & 0 & 1 \\
0 & 0 & -\frac{176}{1525} \\
0 & 0 & -\frac{7}{61}
\end{array}\right), B^{(1)}=\left(\begin{array}{ccc}
-\frac{61}{24} & \frac{125}{24} & -\frac{11}{3} \\
0 & -\frac{468}{305} & \frac{1296}{1525} \\
0 & -\frac{100}{61} & \frac{46}{61}
\end{array}\right)
$$

The first characteristic polynomial of the block method (10) is given by

$$
\begin{aligned}
& \rho(\eta)=\operatorname{det}\left(\eta M^{(1)}-M^{(0)}\right) \\
& =\left|\eta M^{(1)}-M^{(0)}\right| \\
& =0
\end{aligned}
$$

Thus, this leads to

$$
\begin{gathered}
\rho(\eta)=\left|\eta\left(\begin{array}{ccc}
1 & 0 & 0 \\
-\frac{1701}{1525} & 1 & 0 \\
-\frac{68}{61} & 0 & 1
\end{array}\right)-\left(\begin{array}{llc}
0 & 0 & 1 \\
0 & 0 & -\frac{176}{1525} \\
0 & 0 & -\frac{7}{61}
\end{array}\right)\right| \\
=\eta^{3}(\eta-1) \\
\Rightarrow \eta_{1}=1, \eta_{2}=0, \eta_{3}=0
\end{gathered}
$$

Since $\left|\eta_{i}\right| \leq 1, i=1,2 \& 3$, then by [8], the block method (9) is zero stable.

\subsection{Order and Error Constant}

The block hybrid method (9) is of order $\mathrm{p}=3$. Since the block method is zero stable and has order $p>1$, then by [11], the block method (9) is convergent.

\subsection{Stability Region}

The stability of the newly constructed block method is plotted using the method found in [1], [7], [2] and [3] whence the stability function $L(z): \mathbb{C} \rightarrow \mathbb{C}$ which is a rational function with real coefficients for the new block hybrid method (9) using Maple software is given by

$$
L(z)=\frac{-z^{2}-12 z-30}{18 z^{3}-37 z^{2}+48 z-30}
$$


The stability domain R also called stability region of (14) is defined according to [9] as

$$
R=\{z \in \mathbb{C}:|L(z)| \leq 1\}
$$

In Figure 1, the small circles represent the zeros and plus signs represent the poles of (14). The region $\mathrm{R}$ in the complex plane which satisfies (15) for the stability function (14), represents the stability region of the new method.

From figure 1, there are no poles of the stability function $\mathrm{L}(\mathrm{z})$ in the left half of the complex plane, thus (9) is A-stable according to [9].

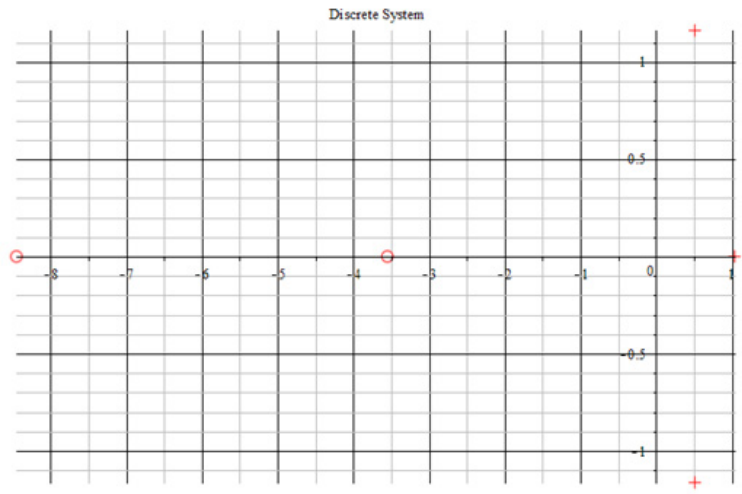

Fig. 1: Plot of stability function L(z) of (14).

\section{Simulation}

Example 1. The solution of the model (2) obtained using (9) in block form and the results obtained by the Matlab ODE $15 \mathrm{~s}$ using the following parameters in example 1 are shown in fig. 2 :

$k_{1}=0.025, k_{2}=0.2, k_{3}=0.4, \mathrm{C}_{a}(0)=1, \mathrm{C}_{b}(0)=0, \mathrm{C}_{c}(0)=0, \mathrm{C}_{d}(0)=0,0 \leq t \leq 100, h=0.01$

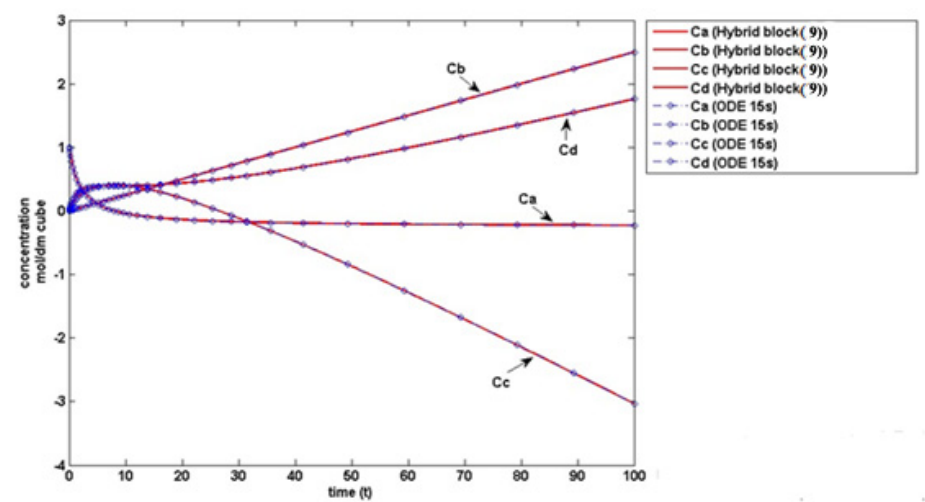

Fig. 2: Solution curves of (2) using the block method (9) and ODE 15s (dotted blue lines). 
Example 2. The solution of the model (2) obtained using (9) in block form and the results obtained by the Matlab ODE 15 s using the following parameters are shown in fig. 3 :

$k_{1}=0.025, k_{2}=0.2, k_{3}=0.4, \mathrm{C}_{a}(0)=120, \mathrm{C}_{b}(0)=0, \mathrm{C}_{c}(0)=0, \mathrm{C}_{d}(0)=0,0 \leq t \leq 100, h=0.01$

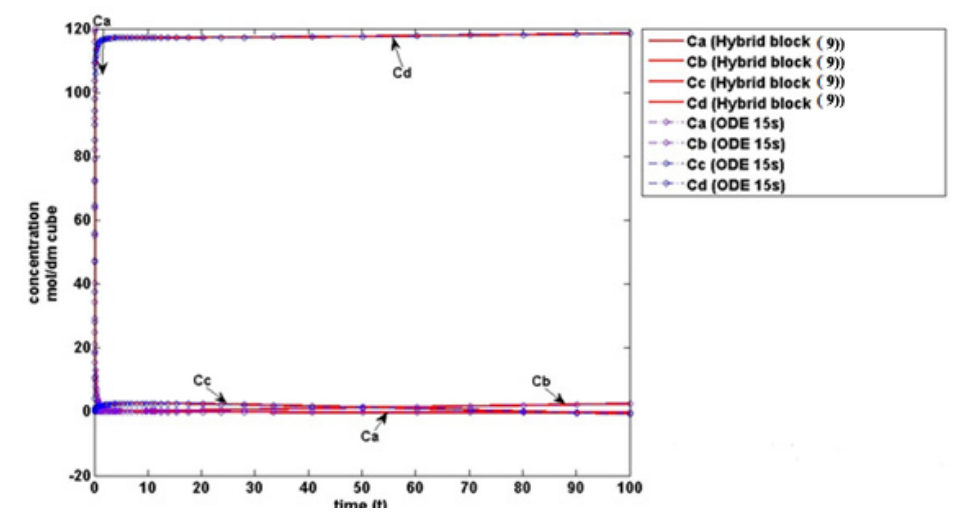

Fig. 3: Solution curves of (2) using the block method (9) and ODE 15s (dotted blue lines).

Example 3. The solution of the model (4) obtained using (9) in block form and the results obtained by the Matlab ODE 23 s using the following parameters are shown in fig. 4 :

$a=1, \mathrm{~b}=5, \alpha=3.10, \in=1, \mathrm{c}=0.2, \mathrm{~d}=5, u(0)=0.2, v(0)=0.1, \mathrm{k}=0.8,0 \leq t \leq 300, h=0.01$

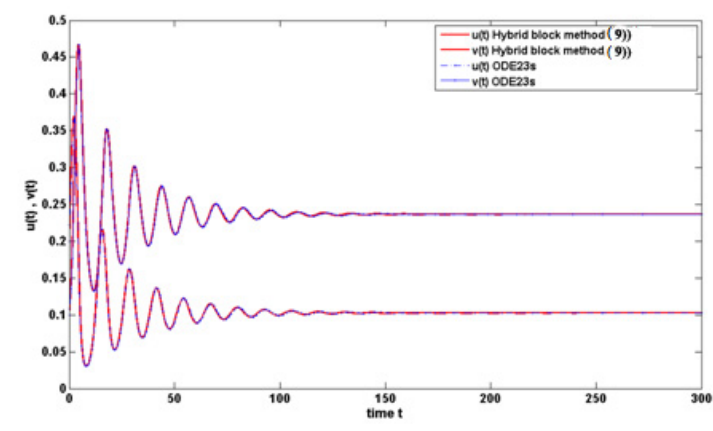

Fig. 4: Solution curves of (4) using the block method (9) and ODE 23s (dotted blue lines).

Example 4. The solution of the model (4) obtained using (9) in block form and the results obtained by the Matlab ODE 23 s using the parameters in example 4 are shown in fig. 5 :

$a=1, \mathrm{~b}=5, \alpha=3.10, \epsilon=1, \mathrm{c}=0.2, \mathrm{~d}=5, u(0)=0.2, v(0)=0.1, \mathrm{k}=0.3,0 \leq t \leq 300, h=0.01$ 


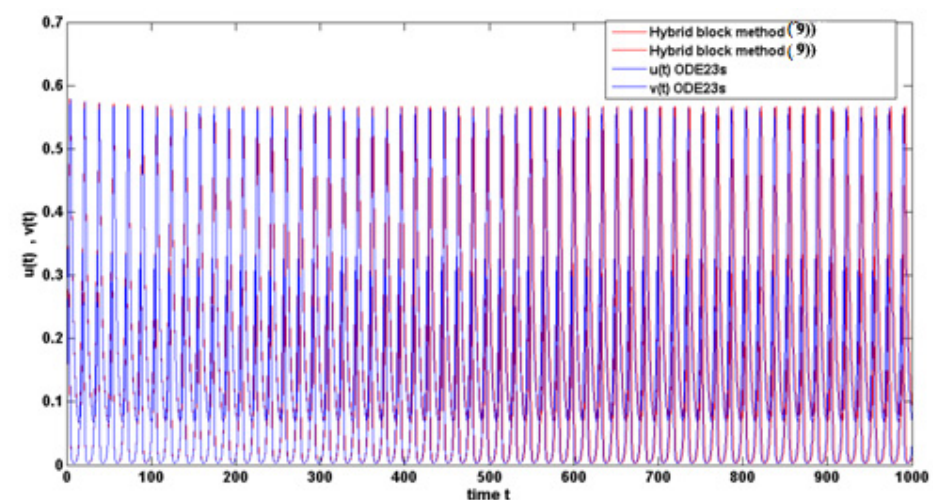

Fig. 5: Solution curves of (4) using the block method (9) and ODE 23s (dotted blue lines).

\section{Discussion}

Comparison of the new block method (9) and ODE 15s (dotted blue lines) shows that they compare well, although the Matlab ODE $15 \mathrm{~s}$ has the advantage of well enhanced variable step size. Figure 2 shows initial concentration $\mathrm{C}_{a}(0)=1.0$ $\mathrm{mol} / \mathrm{dm}^{3}$ of pure A while Figure 3 shows initial concentration $\mathrm{C}_{a}(0)=120.0 \mathrm{~mol} / \mathrm{dm}^{3}$ of pure A. The initial concentration has remarkable effects on the reaction profile as depicted in Figures 2 and 3.

The solutions of Bazykin ecological model (4) as shown in Figures 4 and 5 show that the newly derived block hybrid method (9) also compares well with ODE23s .

\section{Conclusion}

In this paper, we carried out a research on the application of the newly derived block hybrid method (9) incorporating $x=x_{n+\frac{9}{5}}$ as off-step collocation point to solve the Trambouze reactor model and Bazykin ecological model.

The Figures 2, 3, 4 and 5 display that the solutions of the new block method (9) compete favourably with the inbuilt ODE $15 \mathrm{~s}$ and ODE23s. Results show the significant performance of the method and its suitability and efficiency in handling Trambouze reaction schemes and Bazykin ecological model.

\section{Competing interests}

The authors declare that they have no competing interests.

\section{Authors' contributions}

All authors have contributed to all parts of the article. All authors read and approved the final manuscript.

\section{References}

[1] Adedire, O., Sirisena, U.W., Numerical Treatment of Van De Vusse Reactor model using a Three-Step Block HBDF Method, African Journal of Natural Sciences.18, 2015, ISSN: 1119-1104, 49-56.

[2] Akinfenwa, O.A, Jator,S.N and Yao,N.M., A Linear Multistep Hybrid Methods with Continuous Coefficients for Solving Stiff Ordinary Differential Equations, Journal of Modern Mathematics and Statistics, 2011, 5(2), 47-53. 
[3] Akinfenwa, O.A, Jator,S.N and Yao,N.M., On the Stability of Continuous Block Backward Differentiation Formula for Solving Stiff Ordinary Differential Equations, J. of Mod. Meth. in Numer. Math, 2012, 3(2), 50-58.

[4] Alekseev, V.V., Effect of saturation factor on dynamics of predator prey system., Biofizika, 1973, 18, 922-926.

[5] Arino, O., Mikram, J., Chattopadhyay, J., Infection on prey population may act as a biological control in ratio-dependent predatorprey model., Nonlinearity, 2004, 17, 1101-1116.

[6] Bazykin,A.D., Nonlinear Dynamics of Interacting Populations., Series on Nonlinear Science, 1988, Ser.A, vol. 11. World Scientific, Singapore. Chua, L.O. (Ed.), Original Russian version: Bazykin, A.D. Nauka, Moscow (1985).

[7] Fatunla, S.O., Block Methods for Second Order ODEs., International Journal of Computer Mathematics, 1991, 41, 55-63.

[8] Fatunla, S.O., A Class of Block Methods for Second Order IVPs, International Journal Computer and Mathematics, 1994, 55, pp $119-133$

[9] Hairer, E and Wanner, G., Solving ordinary differential Equations II: stiff and differential algebraic problems(second ed), Berlin, Springer-Verlag, ISBN 978-3-540-60452-5.

[10] Haque, M., Ratio-Dependent Predator-Prey Models of Interacting Populations, Bulletin of Mathematical Biology (2009) 71: 430452 DOI 10.1007/s11538-008-9368-4

[11] Henrici, P., Discrete Variable Methods in ODEs, John Wiley, 1962, New York.

[12] Kokossis, A., and Floudas, C., Optimization of complex reaction network 1: Isothermal operation, Chem. Eng.Sci, New York, 45, p. 595-614.

[13] Lambert, J.D., Numerical methods for Ordinary Differential Systems: The Initial Value Problem, John Wiley and Sons, New York, pp.293.

[14] Lie, I and Norsett, S.P., Super Convergence for Multistep Collocation, J.Math Comp, 1989, 52, pp. 65-79

[15] Onumanyi, P., Awoyemi D.O, Jator S.N and Sirisena, U.W., New Linear Multistep Methods with Continuous coefficients for First Order Initial value problems, Journal of Nigeria Mathematical Society, 1994, 13 , pp 27-51

[16] Onumanyi. P, Sirisena, U.W., and Jator S.N., Continuous Finite Difference Approximations for Solving differential Equations, International Journal of Computer Mathematics, 1999, 72, pp. 15-27.

[17] Trambouze, P.J.; Piret, E.L., Continuous stirred tank reactors: designs for maximum conversions of raw material to desired product: homogeneous reactions, AIChE J., 1959, New York, v. 5, p. 384-390.

[18] VanColler,L., Automated techniques for the qualitative analysis of ecological models: Cont-inuous models., Conservation Ecologie [online],1(1):5,(1997);http://www.consecol.org/vol1/iss1/art5/

[19] Wang, X; Wang, C; Jin S;Chen, H, Optimal operating conditions for reactor-separator-recycle system., Journal of Scientific and Industrial Research, 2006, vol. 95, pp. 867-872 